\title{
Migration of cations induces reversible performance losses over day/night cycling in perovskite solar cells
}

Konrad Domanski, ${ }^{1}$ Bart Roose, ${ }^{2}$ Taisuke Matsui, ${ }^{3}$ Michael Saliba, ${ }^{1}$ Silver-Hamill TurrenCruz, ${ }^{4}$ Juan-Pablo Correa-Baena, ${ }^{4}$ Cristina Roldan Carmona, ${ }^{5}$ Giles Richardson, ${ }^{6}$ Jamie M. Foster, ${ }^{6}$ Filippo De Angelis, ${ }^{8,9}$ James M. Ball, ${ }^{10}$ Annamaria Petrozza, ${ }^{10}$ Nicolas Mine, ${ }^{11}$ Mohammad K. Nazeeruddin, ${ }^{5}$ Wolfgang Tress, ${ }^{1}$ Michael Grätzel, ${ }^{1}$ Ullrich Steiner, ${ }^{2}$ Anders Hagfeldt, ${ }^{4}$ Antonio Abate ${ }^{1,2 *}$

${ }^{1}$ Laboratory for Photonics and Interfaces, Institute of Chemical Sciences and Engineering, École Polytechnique Fédérale de Lausanne, CH-1015-Lausanne, Switzerland.

${ }^{2}$ Adolphe Merkle Institute, University of Fribourg, Chemin des Verdiers 4, CH-1700 Fribourg, Switzerland

${ }^{3}$ Advanced Research Division, Panasonic Corporation,1006, (Oaza Kadoma), Kadoma City, Osaka 571-8501, Japan.

${ }^{4}$ Laboratory of Photomolecular Science, Institute of Chemical Sciences and Engineering, École Polytechnique Fédérale de Lausanne, CH-1015-Lausanne, Switzerland.

${ }^{5}$ Group for Molecular Engineering of Functional Materials, Institute of Chemical Sciences and Engineering, École Polytechnique Fédérale de Lausanne, CH-1015-Lausanne, Switzerland.

${ }^{6}$ Mathematical Sciences, University of Southampton, UK (SO17 1BJ)

${ }^{7}$ Department of Mathematics, University of Portsmouth, Portsmouth, UK (PO1 2UP).

${ }^{8}$ Computational Laboratory for Hybrid/Organic Photovoltaics (CLHYO), CNR-ISTM, Via Elce di Sotto 8, 06123 Perugia, Italy.

${ }^{9}$ CompuNet, Istituto Italiano di Tecnologia, Via Morego 30, 16163 Genova, Italy.

${ }^{10}$ Center for Nano Science and Technology@Polimi, Istituto Italiano di Tecnologia, via Giovanni Pascoli 70/3, 20133 Milan, Italy

${ }^{11}$ Laboratory for Nanoscale Materials Science, Empa, Swiss Federal Laboratories for Material Science and Technology, Ueberlandstr. 129,8600 Duebendorf, Switzerland

*Corresponding author: AA antonioabate83@gmail.com Antonio.abate@unifr.ch 
Keywords: Perovskite solar cell, hysteresis, photovoltaics, ionic migration, perovskite stability, ToF-SIMS, depth-profile, ageing perovskite solar cells 


\begin{abstract}
Perovskites have been demonstrated in solar cells with power conversion efficiency well above $20 \%$, which makes them one of the strongest contenders for the next generation photovoltaics. While there are no concerns about their efficiency, very little is known about their stability under illumination and load. Ionic defects and their migration in the perovskite crystal lattice are one of the most alarming sources of degradation, which can potentially prevent the commercialization of perovskite solar cells (PSCs). In this work, we provide direct evidence of electric field-induced ionic defect migration and we isolate their effect on the long-term performance of state-of-the-art devices. Supported by modelling, we demonstrate that ionic defects, migrating on timescales significantly longer (above $10^{3} \mathrm{~s}$ ) than what has so far been explored (from $10^{-1}$ to $10^{2} \mathrm{~s}$ ), abate the initial efficiency by $10-15 \%$ after several hours of operation at the maximum power point. Though these losses are not negligible, we prove that the initial efficiency is fully recovered when leaving the device in the dark for a comparable amount of time. We verified this behaviour over several cycles resembling day/night phases, thus probing the stability of PSCs under native working conditions. This unusual behaviour reveals, that research and industrial standards currently in use to assess the performance and the stability of solar cells need to be adjusted for PSCs. Our work paves the way towards much needed new testing protocols and figures of merit specifically designed for PSCs.
\end{abstract}




\section{Introduction}

Perovskite solar cells (PSCs) have the potential to become a new generation of photovoltaics with the shortest energy payback time and lowest $\mathrm{CO}_{2}$ emission factor among existing technologies. ${ }^{1}$ In only a few years, an unprecedented progress in preparation procedures and material compositions has delivered lab-scale devices that have now reached power conversion efficiencies (PCEs) of up to $22.1 \%{ }^{2}$. However, this impressive improvement of the PCE has not been matched by an equal advancement in the knowledge of the performance losses under standard working conditions (illumination and load). ${ }^{3-7}$

So far, discussion around PSCs stability has mainly focused on oxygen, ${ }^{8}$ water ${ }^{5}$ and UV light exposure $^{9}$ as causes of rapid performance degradation in PSCs. These extrinsic factors have been associated with a number of degradation mechanisms that can be retarded using the sealing technologies industrialised for organic electronics, which provide oxygen and humidity barriers and protection against UV light. ${ }^{10,11}$ Conversely, prolonged exposure to solar cell operational temperatures (above $50^{\circ} \mathrm{C}$ ) can cause severe degradation, which cannot be avoided by sealing the PSCs. These, so called intrinsic losses, have been mostly associated with the degradation of organic materials and metal contacts within PSCs. ${ }^{3,12,13}$ Indeed, significant progress has been made by replacing the organic components with their inorganic counterparts and passivating the interfaces between the different layers composing the device. $^{13-18}$ Nonetheless, temperature activated formation and migration of ionic defects within the organic-inorganic $\mathrm{ABX}_{3}$ perovskite lattice remains a potential source of instability for perovskite photovoltaics. ${ }^{19-22}$ Halide anion $(\mathrm{X})$ vacancies have been calculated to show the lowest formation energies, ${ }^{23}$ with bromide vacancies being favoured over iodide. ${ }^{24}$ Correspondingly, $\mathrm{X}$ vacancies (together with interstitial $\mathrm{X}$ ) have been shown to be the most mobile defects, followed by cation A and B vacancies. ${ }^{25-27}$ Several studies indicated that, regardless of particular architecture and constituents within the PSCs, X defects migrate and reversibly accumulate within the perovskite lattice in narrow Debye layers at the interfaces with the charge selective contacts. ${ }^{19,28-34}$ Depending on voltage and light bias conditioning, accumulation of ions (and their vacancies) partially screens the built-in electric field and possibly creates interfacial electronic trap states, which reduce the charge extraction efficiency. $^{25,30,31,34-43}$ Ion migration on timescales from $10^{-1}$ to $10^{2} \mathrm{~s}$ has been widely investigated to explain the hysteresis of current density-voltage $(J-V)$ curves. $^{36,37,40,44-48}$ However, the impact of $\mathrm{X}$ and potentially $\mathrm{A}$ and/or B defect formation and migration on PSC performance on timescales above $10^{3} \mathrm{~s}$, which are indicative of long-term stability, remains unknown. ${ }^{49}$ Little experimental evidence exists on this subject since separating reversible ion 
migration from any non-reversible long-term degradation is complex in real device working conditions, i.e. prolonged exposure to continuous light and voltage bias. ${ }^{29,39,50-55}$

In this work, we provide direct evidence of electric field-induced ion migration and its effects on the long-term performance of perovskite solar cells working under different loads. Cooling in situ the active area of working PSCs, we are able to inhibit thermally induced, non-reversible degradation, thereby exposing fully reversible performance losses. Within several hours of operation at the maximum power point (MPP), the reversible losses abate a significant fraction of the initial PCE, which is followed be a period of stabilization. Supported by modelling and elemental depth profiling, we correlate the reversible performance losses in PSCs to the migration of ion vacancies on timescales (above $10^{3} \mathrm{~s}$ ), which are significantly longer than those explored so far (from $10^{-1}$ to $10^{2} \mathrm{~s}$ ). These unusually slow dynamics reveal that academic and industrial standards currently in use to assess the performance and stability of solar cells need to be adjusted for PSCs, which exhibit phenomena previously unknown to the photovoltaics community. Importantly, we show that over natural day/night cycles, PSCs that reversibly degrade during the day recover overnight to "start fresh" every morning. Our work paves the way towards developing specific testing protocols, definition of new figures of merits and calculation of energy payback time that are needed to characterize PSCs. 


\section{Results and discussion}

a

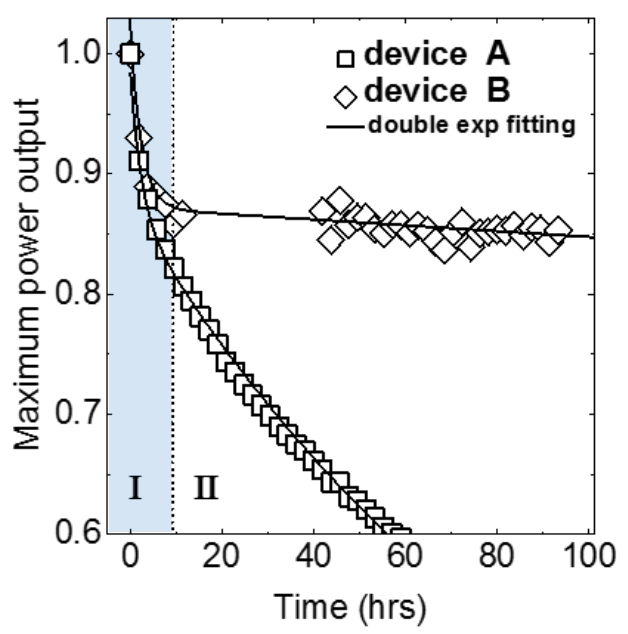

b
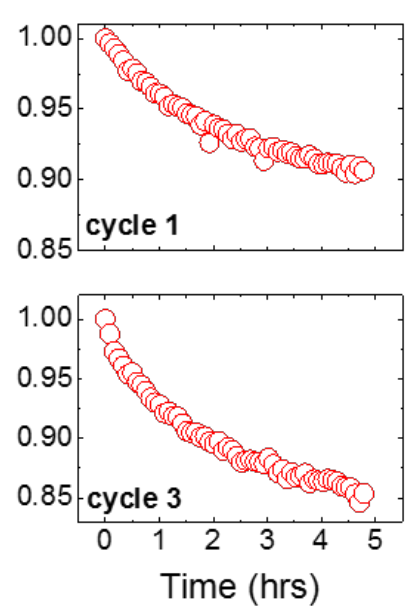

device C
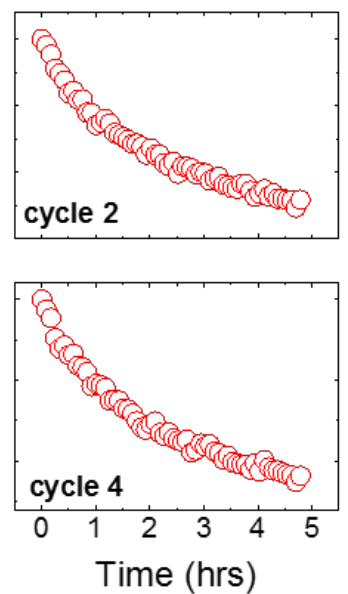

C

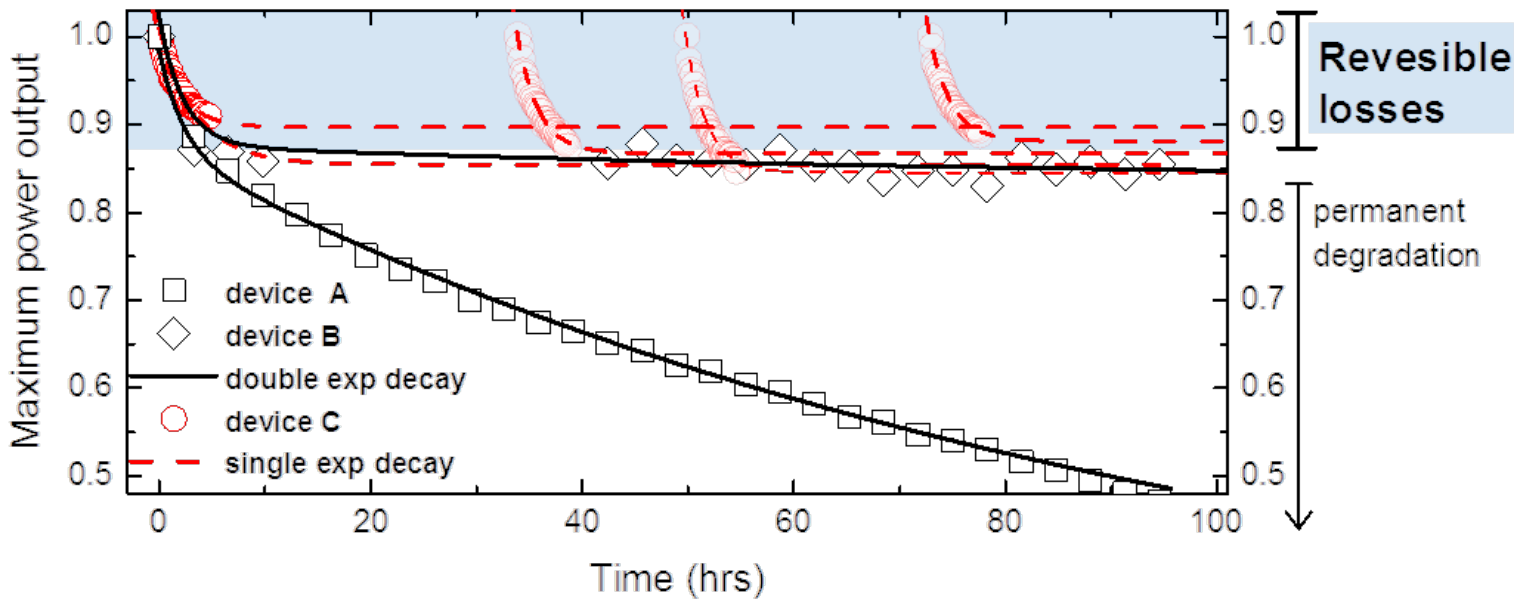

Figure 1. Maximum power output tracking for 3 identically prepared perovskite solar cells (device A, B and C) measured under UV-filtered 1 sun equivalent light. Devices were continuously kept at the maximum power point by the standard "perturb and observe" method. The efficiency of all freshly made devices was above $20 \%$ (see SI) and it dropped to around $17-18 \%$ after 2-3 weeks, when the stability data were recorded. Data were normalized to the maximum value, which was usually reached within several minutes of tracking. a Devices A and B were continuously tracked for over 100 hours. b Device C was cyclically tracked 4 times for 5 hours and it was left in dark at open circuit in between the consecutive measurements. c Experimental data were fitted to an exponential decay (single or double) and the fitting parameters are reported in Table 1.

Table 1. Fitting parameters of the exponential decay functions used in Figure 1. Device A and $\mathrm{B}$ curves were fitted with a double exponential decay function $J=A_{1} \exp \left(-x / t_{1}\right)+A_{2} \exp (-$ $\left.x / t_{2}\right)+J_{0}$, where $t$ is the time constant, $J_{o}$ is the residual power output and $A$ is the preexponential factor. Device $\mathrm{C}$ curves were fitted with a single exponential decay function $J=$ $A_{1} \exp \left(-x / t_{1}\right)+J_{0}$.

\begin{tabular}{ccc}
\hline & $\begin{array}{c}\boldsymbol{t}_{\mathbf{1}} \\
(\text { hrs })\end{array}$ & $\begin{array}{c}\boldsymbol{J}_{\mathbf{0}} \\
\text { (residual power \%) }\end{array}$ \\
\hline Device A & $2.3 \pm 0.2$ & $25 \pm 1$ \\
Device B & $2.3 \pm 0.2$ & $84 \pm 2$ \\
Device C, cycle 1 & $2.1 \pm 0.05$ & $90 \pm 0.1$
\end{tabular}




$\begin{array}{lll}\text { Device C, cycle 2 } & 2.0 \pm 0.04 & 87 \pm 0.1 \\ \text { Device C, cycle 3 } & 1.9 \pm 0.04 & 85 \pm 0.1 \\ \text { Device C, cycle 4 } & 2.0 \pm 0.04 & 88 \pm 0.1\end{array}$

To study the impact of the long-term ion migration on device performance and stability, we prepared state-of-the-art PSCs, using the mixed halide-cation perovskite composition $\mathrm{CH}_{3} \mathrm{NH}_{3} / \mathrm{CH}\left(\mathrm{NH}_{2}\right)_{2} \mathrm{~Pb} \mathrm{Br} / \mathrm{I}$ and the antisolvent deposition method on mesoporous $\mathrm{TiO}_{2}$ substrates, ${ }^{56}$ which enabled the realization of power conversion efficiencies above $20 \%$ (see device characterization in SI). We tracked the maximum power output of 3 identically prepared devices (device A, B and C in Figure 1). During the experiment, the devices were kept under 1 sun-equivalent white LED illumination at MPP (around $0.85 \mathrm{~V}$ ) and under $\mathrm{N}_{2}$ atmosphere. The devices A and B were continuously tracked for over 100 hours. As previously reported, ${ }^{3,7,13}$ the maximum power output traces can be described with a double exponential decay function showing an initial rapid (I in Figure 1a) and a subsequent slower decay regime (II in Figure 1a). 3, 73 We have previously shown that the latter (regime II) is due to degradation involving one or more device components, while the early decay (regime I) has remained unexplained so far and it will be the main object of this study. ${ }^{3,7}$ We selected two devices exhibiting very different II regimes: a rather unstable device A (residual power output $J_{0}=25 \%$, Table 1 ) and device B showing particularly good stability (residual power output $J_{0}=84 \%$, Table 1). Since the long-term degradation is a convolution of several mechanisms that may abruptly impact the performance, ${ }^{57}$ it is not surprising that identically prepared devices age differently. However, it is rather unexpected that they have identical time constants for the decay regime I ( $t_{1}$ in Table 1$)$. To isolate regime I from the subsequent degradation (regime II), the maximum power point tracking (MPPT) for a device C was stopped after only 5 hours and repeated periodically after leaving the device resting in dark for a varying number of hours (Figure 1b). Surprisingly, the initial power output at each cycle was similar or even slightly higher to the previous one, demonstrating that the initial performance losses are fully reversible (regime I) and are thus separated from the subsequent permanent degradation (regime II). The decay traces and exponential fits of devices A, B and $\mathrm{C}$ are summarized in Figure 1c. The residual power output for device $\mathrm{C}$ at each cycle (dashed line in Figure 1c, $J_{0}$ in Table 1) lies between 0.85 and 0.9 , similar to what was extracted for the more stable device B after 100 hours of MPPT. This confirms that the performance losses in device $\mathrm{B}$ are mostly reversible, with a marginal contribution of permanent degradation. We repeated this experiment for devices with planar and inverted architectures, varying the 
electron and hole selective contact materials (see SI), with similar conclusions. While we collected data for PSCs prepared with mixed halide-cation perovskite, reversible losses have been observed for a broad range of perovskite compositions. ${ }^{18,52-54,58}$ Therefore, we confidently conclude that the reversible losses are intrinsic to the perovskite as photovoltaic material and not to specific material composition or device architecture.

It is worth noting that the characteristic times for the reversible degradation/recovery are representative of the native working conditions for solar cells, i.e. day/night cycles. Therefore, a PSC producing energy during the day will have time to recover during night to "start fresh" every morning. This finding has an important practical implication for establishing industrial benchmarks for ageing and cost/operation time calculations of PSCs. 
a Control and biased pixel both heated at $70^{\circ} \mathrm{C}$

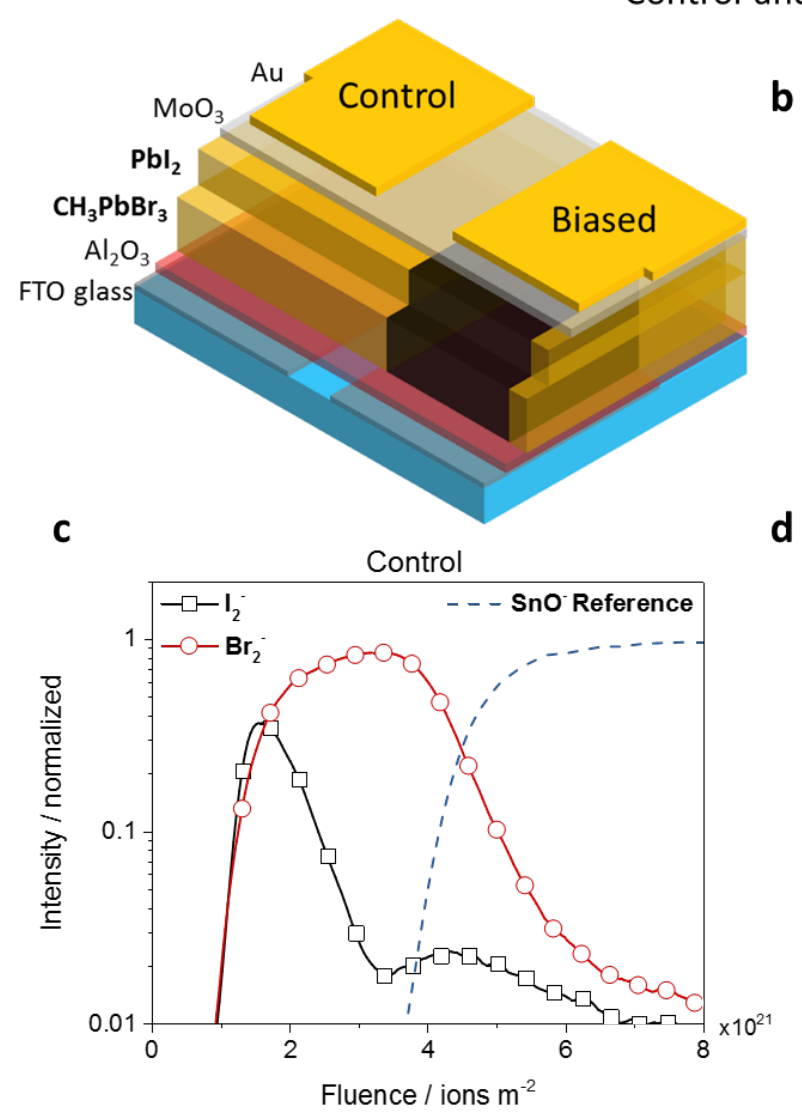

b

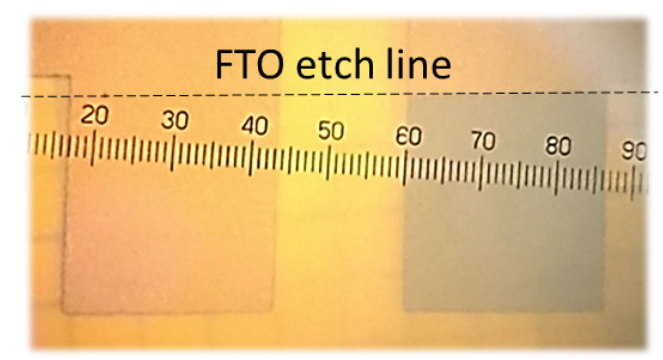

d

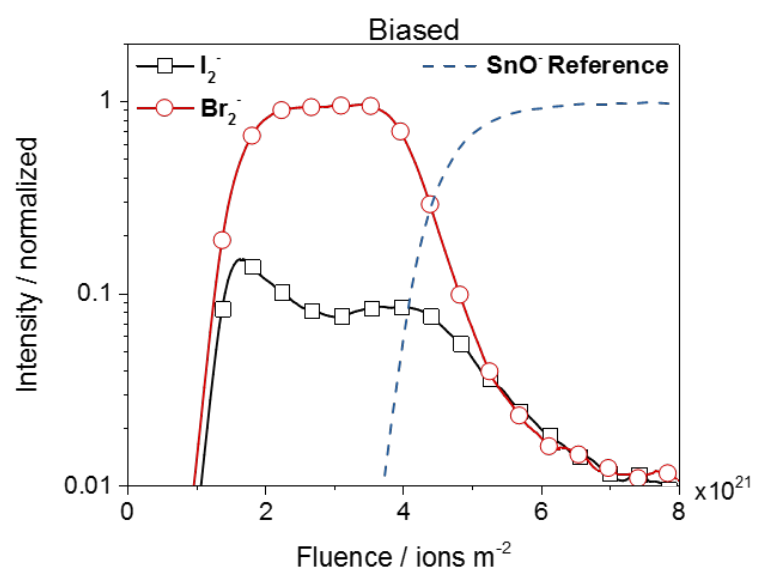

Figure 2. a Schematics of the sample prepared for the biasing experiment, consisting of two electronically-separated pixels on the same substrate. Both pixels were heated to $70{ }^{\circ} \mathrm{C}$ in $\mathrm{N}_{2}$ atmosphere. Only one pixel was cyclically biased at +2 and $-2 \mathrm{~V}$ every 30 minutes for 16 hours (biased pixel). b Top view image of the sample after the biasing (scale in millimetre). Time of flight secondary ion mass spectrometry (ToF-SIMS) depth profiles of the control (c) and biased (d) pixels showing the relative secondary ions intensity of iodine and bromine clusters across the film depth. The $\mathrm{SnO}^{-}$signals coming from the fluorine doped $\mathrm{SnO}_{2}(\mathrm{FTO}$ glass) are used as a reference, to which all traces in the respective graph are normalized.

Reversible performance degradation as a result of light soaking has been previously investigated by Bag et al. ${ }^{52}$ and Nie et al. ${ }^{54}$ In both the works, the authors concluded the heat from the sunlight is responsible for lattice strain and consequent defect formation. In contrast to existing literature, we studied the reversible losses under real device working conditions, i.e. prolonged exposure to continuous light and voltage bias around MPP. We postulated that the ions and ion defects migration are the cause of reversible losses. In order to provide direct evidence of ion migration, we deposited a $\mathrm{PbI}_{2}$ layer on top of a $\mathrm{CH}_{3} \mathrm{NH}_{3} \mathrm{PbBr}_{3}$ perovskite layer in capacitor-like devices, as represented in the schematic in Figure 2a. These devices were heated to $70{ }^{\circ} \mathrm{C}$ in a nitrogen atmosphere and one of the pixels was cyclically biased at 
+2 and $-2 \mathrm{~V}$ every 30 minutes (leakage current remained below $\pm 1 \mathrm{~mA} \mathrm{~cm}{ }^{-2}$ ). After 16 hours, we found that the biased pixel had changed its colour to black, while the unbiased pixel (also kept at $70{ }^{\circ} \mathrm{C}$ ) remained yellow, as clearly visible in Figure $2 \mathrm{~b}$. Notably, the colouring of the biased pixel accurately follows the overlap between the top (gold) and the bottom (FTO) electrodes. This suggests that, in response to the electric field, ions migrate between the yellow $\mathrm{CH}_{3} \mathrm{NH}_{3} \mathrm{PbBr}_{3}$ and $\mathrm{PbI}_{2}$ layers to form the black $\mathrm{CH}_{3} \mathrm{NH}_{3} \mathrm{PbI}_{x} \mathrm{Br}_{(3-x)}$ perovskite. We made use of time of flight secondary ion mass spectrometry (ToF-SIMS) to measure the effective elemental changes within the layers. ${ }^{59}$ From the $\mathrm{I}_{2}^{-}, \mathrm{Br}_{2}{ }^{-}$elemental depth profiles shown in Figure 2c and d, we found that iodine and bromine distributions are significantly changed in the biased pixel compared to the control one. In particular, we observe strong halide mixing, which results in formation of black $\mathrm{CH}_{3} \mathrm{NH}_{3} \mathrm{PbI}_{\mathrm{x}} \mathrm{Br}_{(3-\mathrm{x})}$. This constitutes a strong direct evidence that halides can indeed migrate within perovskite driven by an electric field and not due to thermal activation or electric current. ${ }^{52,60-62}$

We also analysed the ToF-SIMS depth profiles looking for an indication of organic cation diffusion (by tracing $\mathrm{CN}^{-}$signal). Unfortunately, the depth profiles did not qualitatively correspond to the expected $\mathrm{CN}^{-}$distribution in the control sample (see Figure in $\mathrm{SI}$ ) and hence we deemed the analysis inconclusive (we also found a large amount of organic contamination on the surface of the sample). Additionally, we performed energy-dispersive x-ray (EDX) spectroscopy on cross-section of the same device (see Figure in SI). However, again we could not conclude if cations are mobile, largely due to the fact that the technique is not suited for tracing lightweight elements constituting $\mathrm{CH}_{3} \mathrm{NH}_{3}{ }^{+}$ions (the fact that the devices had to be coated with $\mathrm{C}$ prior to the analysis, made the analysis even more challenging). Given that it is not trivial to provide a direct evidence of organic cation migration, we made use of electric measurements supported by modelling to prove it indirectly. 

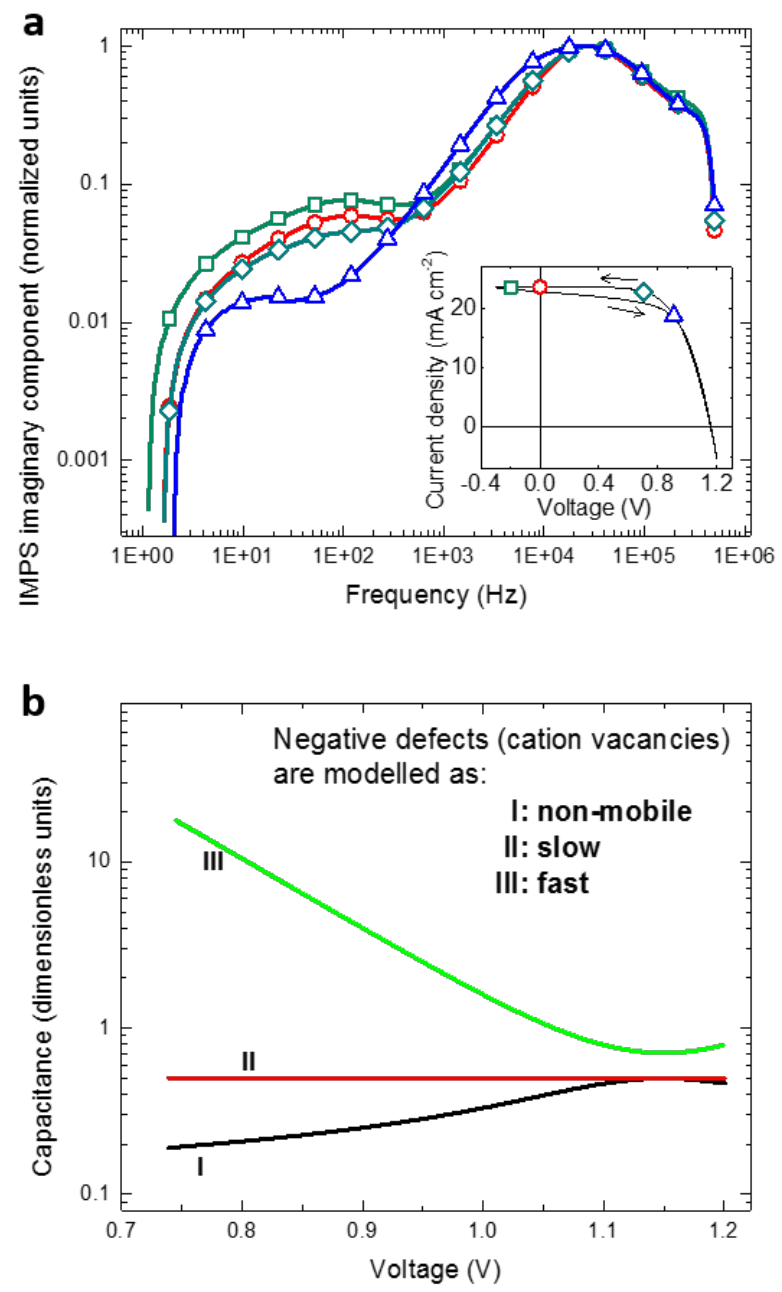

Figure 3. a Imaginary component frequency spectra of the current response to the light modulated $(10 \%$ of the stationary value $) \sim 1$ sun equivalent intensity under different applied voltage biases. Data were normalized to the maximum value corresponding to the peak between 10 and $100 \mathrm{kHz}$. The inset shows the $J-V$ curve recorded for the same devices at 100 $\mathrm{mV} \mathrm{s}^{-1}$ scan rate. b Model calculation of the capacitance per unit surface area at the edge of the perovskite layer as a function of the forward applied voltage bias, assuming the negative defects (cation vacancies) as non-mobile, slow or fast, compared to the timescale of the spectroscopic oscillations in the low frequency region $\left(10^{-1}-10^{3} \mathrm{~Hz}\right)$.

We used intensity modulated photocurrent spectroscopy (IMPS) to monitor ion migration during device operation at the voltages highlighted in the $J$ - $V$ curve in the inset in Figure $3 \mathrm{a}^{63}$ The photocurrent was allowed to settle at each voltage for $300 \mathrm{~s}$ before the frequency scan. Figure 3a shows the imaginary component of the IMPS frequency spectra. The traces show two features in the high frequency region, which we assign to the resonant frequencies of the charge dynamics within the perovskite layer (above $10^{5} \mathrm{~Hz}$ ) and the electron/hole charge selective materials (between $10^{4}-10^{5} \mathrm{~Hz}$ ). ${ }^{64}$ The spectra are similar in this region, which suggests that charge transport is not significantly affected by the voltage applied. An 
additional feature at around $10^{2} \mathrm{~Hz}$ is clearly visible in the low frequency region. We have previously associated the $10^{-1}-10^{3} \mathrm{~Hz}\left(\right.$ or $10^{-3}-10 \mathrm{~s}$ ) resonant frequency window with the ion defect migration within the perovskite crystal lattice. ${ }^{64,} 65$ The migration and accumulation of ionic defects at the interfaces with the charge selective contacts results in the formation of two narrow Debye layers (DLs). ${ }^{34}$ The qualitative relation between the DL capacitance and the applied voltage was recently derived from the drift diffusion model proposed by Richardson et al. ${ }^{34}$ Different from the previous report, here we are taking into account processes occurring on both the relatively short timescale of the spectroscopic oscillations $\left(10^{-3}-10 \mathrm{~s}\right)$ and on the longer settling time $(300 \mathrm{~s})$ before the start of the frequency scan (Figure 3b, see SI for more details). Based on the calculations of activation energies for migration of different ionic defects in $\mathrm{CH}_{3} \mathrm{NH}_{3} \mathrm{PbI}_{3}$, negatively-charged cation

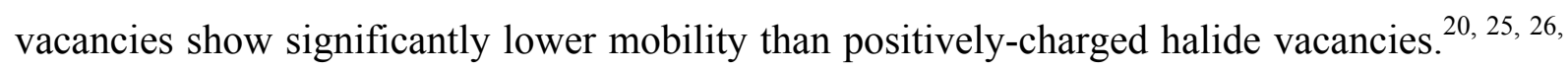
66-68 Given that cation vacancies are slower than the halide ones, three different scenarios are possible: (I) only halide vacancies are mobile on both spectroscopic $\left(10^{-3}-10 \mathrm{~s}\right)$ and settling (300 s) timescales, while all the other defects remain effectively frozen; (II) cation vacancies are sufficiently mobile to equilibrate within the DLs during the settling time, but they are effectively immobile on the spectroscopic timescale; and (III) both halide and cation vacancies are sufficiently mobile to equilibrate within the DLs on spectroscopic timescale. We found that the experimental trend in Figure 3a was best reproduced by the model that accounts for halide vacancy migration, but does not directly account for the slowly moving cation vacancies even on the 300 s settling timescale. This observation suggests that if cation vacancies are effectively mobile, they should migrate over a timescale of hours. 

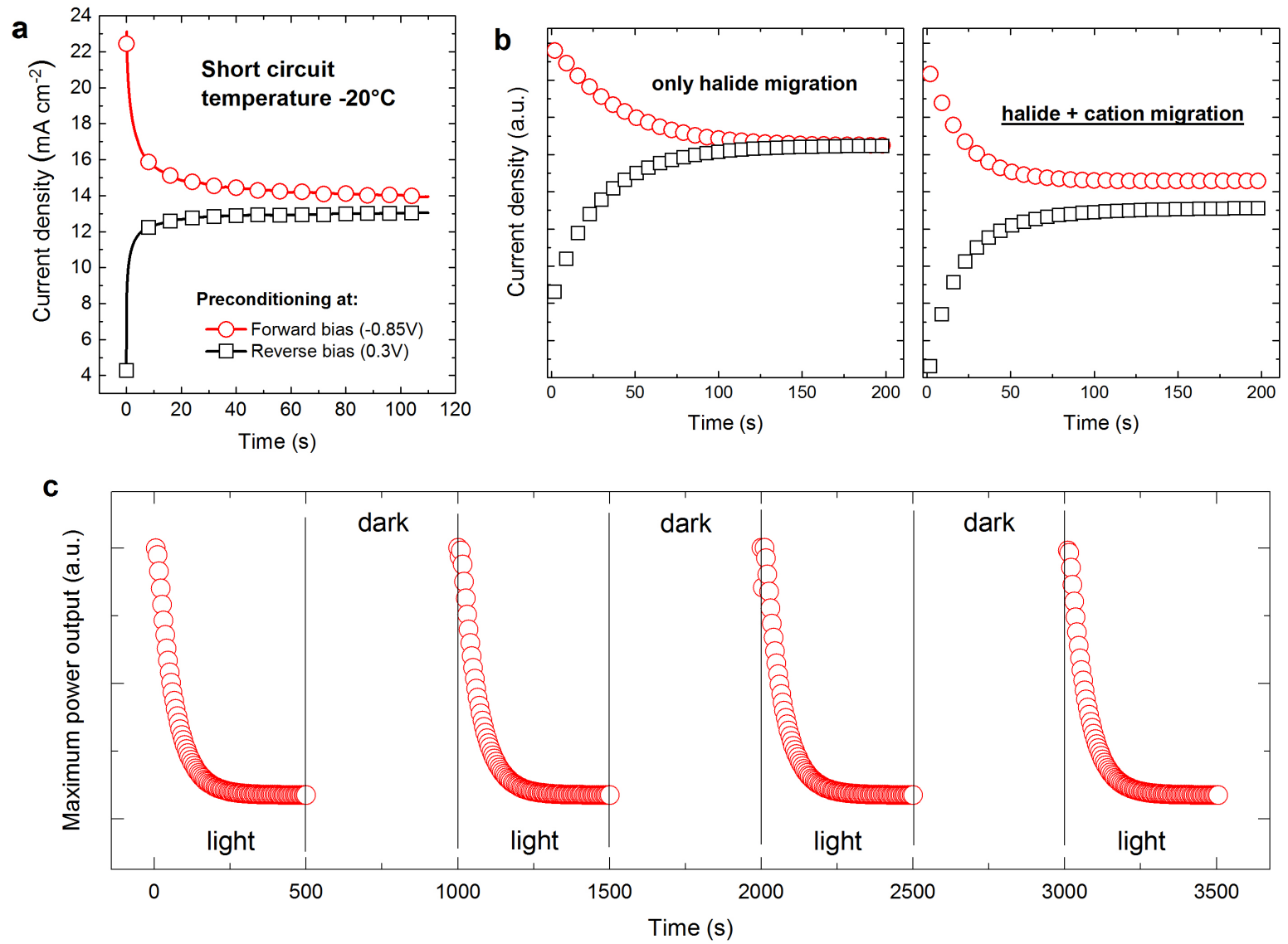

Figure 4. a Current transient dynamics collected from the same device pre-condition for about 20 minutes under forward $(0.85 \mathrm{~V})$ or reverse $(-0.3 \mathrm{~V})$ bias and cooled to $-20{ }^{\circ} \mathrm{C}$ before to abruptly switch to short circuit condition. b Modelled current transient dynamics as predicted by the evolution of the potential (left panel - considering only halide vacancy migration, and right panel - considering fast halide and slow cation vacancy migration). c Modelled maximum power conversion efficiency (with fast halide and slow cation vacancy migration) over 4 cycles of light and dark shows similar (non-quantitative) reversible performance losses to the experiment in Figure 1.

To provide evidence that cation vacancies are effectively mobile we measured the current transient dynamics at short circuit after preconditioning the device at either forward $(0.85 \mathrm{~V})$ or reverse $(-0.3 \mathrm{~V})$ bias (Figure $4 \mathrm{a})$. Under each biasing condition, we waited for the current to stabilize for about 20 minutes at $20{ }^{\circ} \mathrm{C}$, before cooling the device to $-20{ }^{\circ} \mathrm{C}$ and switching it abruptly to short circuit condition. By cooling down the device, we aimed to retard ion migration and capture the transient dynamics resulting from the initial non-equilibrium ion distribution. Following forward bias preconditioning, the short circuit current $\left(\mathrm{J}_{\mathrm{sc}}\right)$ rapidly decreased from over $23 \mathrm{~mA} \mathrm{~cm}$ ch $^{-2}$ towards a value of around $14 \mathrm{~mA} \mathrm{~cm}$. In contrast, subsequent to preconditioning at reverse bias, $\mathrm{J}_{\mathrm{sc}}$ rises from $4 \mathrm{~mA} \mathrm{~cm}^{-2}$ to around $13 \mathrm{~mA} \mathrm{~cm}^{-2}$. Notably, in these two experiments $J_{\mathrm{sc}}$ does not relax to the same value over the 100 s over which the transient is measured; there is a secondary slow timescale process which we 
attribute to the slow motion of cation vacancies. In order to back up this assertion we calculate the short circuit current based on (I) a model of the perovskite layer in which only halide vacancies are mobile (Figure 4b, left-hand panel) and (II) based on a model in which both halide and cation vacancies are mobile (Figure $4 \mathrm{~b}$, right-hand panel) but where cations vacancies move 100 times more slowly than halide vacancies (see SI for further details). It can be seen from Figure 4 that while the model with immobile cation vacancies is able to qualitatively predict the initial part of the transients, it fails to capture the slow timescale decay. However, the inclusion of slow moving cation vacancies into the model (Figure 4b, right-hand panel) gives a picture closer to the reality (Figure 4a) in which there is an initial rapid transient, over tens of seconds, followed by a much longer timescale decay.

In order to investigate the conjecture that the reversible decay in efficiency shown in Figure 1 (which occurs over a timescale of around 1 hour) is due to the slow motion of cation vacancies we simulate the current produced by the device as the light is switched on and off a number of times, the results are shown in Figure $4 \mathrm{c}$ and qualitatively reproduce the behaviour seen in Figure 1c for device $\mathrm{C}$ (plotted in red circles). We conclude by remarking that slow cation vacancy motion thus provides a theory that is capable of explaining both the slow timescale decay in transient short circuit current measurements (Figure 4a) and the reversible decay in efficiency observed in PSCs after prolonged exposure to the light (Figure 1c). 


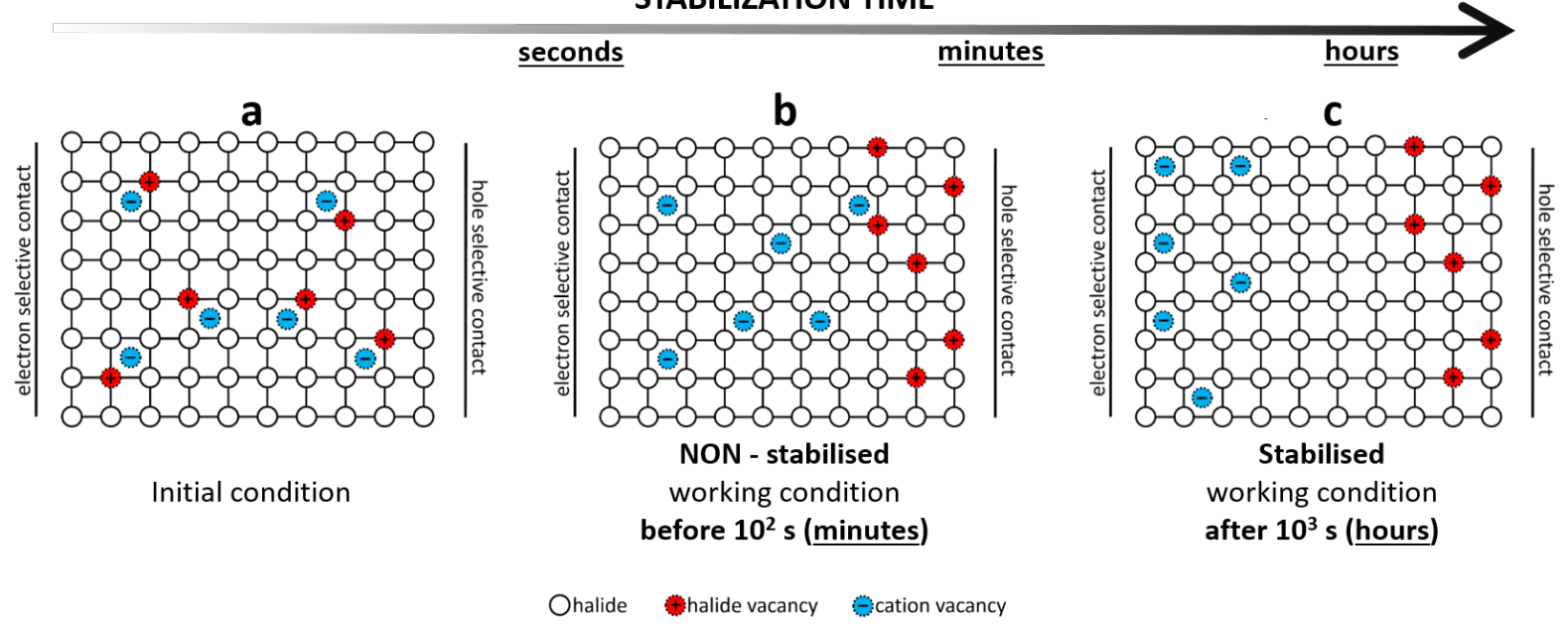

Figure 5. Schematics of the evolution of the ion distribution within the perovskite layer sandwiched between the electron and hole selective contacts under solar cell working conditions: a initial condition, $\mathbf{b}$ non-stabilized condition on the timescale of minutes and $\mathbf{c}$ the stabilized condition on the timescale of hours.

To summarize, we have observed that PSCs degrade reversibly on the timescale of hours regardless of device architecture. We have also proven, that both halide and cation vacancies are mobile (albeit the latter are considerably slower than the former) and their distribution in the perovskite layer can considerably affect charge extraction and, in consequence, PCE of the device. Figure 5 shows a simplistic schematic to condense the results of the experiments and model calculations discussed here. The images represent the halide lattice within the perovskite with ionic vacancies. The initial condition (a) describes a stoichiometric amount of anion and cation vacancies, which are randomly distributed in the perovskite lattice as proposed by Walsh et al. ${ }^{69}$ (b) up to $10^{2} \mathrm{~s}$ (i.e. minutes) after the device was exposed to light and switched to MPP, halide vacancies migrate to form a Debye layer at the interface with hole selective contact, leaving the relatively immobile cation vacancies behind. (c) for timescales longer than $10^{3} \mathrm{~s}$ (i.e. hours), cation vacancies form an additional Debye layer at the interface with the electron selective contact, which in turn inhibits charge extraction from the device. Hence we come back to the original experiment shown in Figure 1. When PSCs are exposed to real operating conditions, the slow cation migration is responsible for the reversible losses in the device on the timescale of hours. However, when the device is given several hours to recover in the dark, the ionic distribution returns to the initial state and the device appears as "fresh". The strong asymmetry in the activation energy for migration of halide and cation vacancies implies that stabilization times on the order of hours (not minutes as widely believed) are required for PSCs to reach a true steady-state working conditions. 
This implies, that traditional solar cell characterization methods, such as measurements of standard $J-V$ curves and incident photon to current efficiency (IPCE) spectra, are usually performed on non-stabilized devices. We propose the maximum power point tracking for several hours as the most reliable method to determine the initial efficiency and the stability of PSCs. 


\section{Conclusions}

In this work, we investigated the impact of the ionic defect migration on the performance and stability of state-of-the-art perovskite solar cells (PSCs). We provide direct evidence of halide migration within the perovskite as a result of applied electric field. In line with previous reports, we found that halide vacancies migrate and accumulate at the interface with the hole selective contact on timescales between $10^{-1}$ and $10^{2} \mathrm{~s}$. The accumulation of interfacial halide vacancies increases as the applied voltage moves from forward to reverse bias, which results in the occurrence of the well-known hysteresis in the current densityvoltage curves of PSCs. In addition to what has been explored previously, we propose that cation vacancies migrating on significantly longer timescales (above $10^{3} \mathrm{~s}$ ) than halide vacancies play a key role in the long-term performance of PSCs. We show, that the accumulation of cation vacancies at the electron contact induces reversible performance losses that abate the initial efficiency of state-of-the-art PSCs by about $10-15 \%$ over several hours of operation at the maximum power point. Although these losses are not negligible, the initial efficiency is fully recovered when leaving the device in dark for a comparable amount of time. We verified this behaviour for devices with different architectures over several cycles resembling day/night phases. We show that PSCs recover their initial efficiency during the night and deliver, every morning, the same efficiency as freshly made devices. This unusual behaviour reveals that research and industry standards currently in use to assess the performance and the stability of solar cells need to be reconsidered for PSCs. Our work provides indications for the much needed new testing protocols and figures of merits specifically designed for PSCs. 


\section{Methods section}

General Methods. All the chemicals were purchased from commercial sources and used without further purification. All the solvents were anhydrous and high purity grade.

Solar cells fabrication. Devices were fabricated on fluorine doped tin oxide (FTO) coated glass substrates. The substrates were cleaned sequentially with Hellmanex in ultrasonic bath for $30 \mathrm{~min}$, then washed with acetone, isopropanol and finally cleaned with oxygen plasma for $5 \mathrm{~min}$. About $30 \mathrm{~nm} \mathrm{TiO}_{2}$ compact layer was deposited on about $150 \mathrm{~cm}^{2}$ of FTO via spray pyrolysis at $450^{\circ} \mathrm{C}$ from a precursor solution prepared with $0.4 \mathrm{~mL}$ of acetyl acetone (Aldrich), $0.6 \mathrm{ml}$ of titanium diisopropoxide bis(acetylacetonate) solution (Aldrich, $75 \%$ in 2propanol) and $9 \mathrm{~mL}$ of ethanol. After the spraying, the substrates were left at $450^{\circ} \mathrm{C}$ for 5 min, then they were slowly cooled down to room temperature. Mesoporous $\mathrm{TiO}_{2}$ layer was deposited by spin coating for $10 \mathrm{~s}$ at $4000 \mathrm{rpm}$ with a ramp of $2000 \mathrm{rpm} \mathrm{s}^{-1}$, using $30 \mathrm{~nm}$ particle paste (18NR-T Dyesol) diluted in ethanol to achieve about $150 \mathrm{~nm}$ thick layer. After the spin coating, the substrate was immediately dried at $100^{\circ} \mathrm{C}$ for $10 \mathrm{~min}$ and then sintered again at $500^{\circ} \mathrm{C}$ for 30 min under dry air flow. Mesoporous $\mathrm{TiO}_{2}$ was doped with lithium by spin coating a $0.1 \mathrm{M}$ solution of Li-TFSI in acetonitrile at $3000 \mathrm{rpm}$ for $30 \mathrm{~s}$. After the spin coating, the substrate was dried at $100^{\circ} \mathrm{C}$ for $10 \mathrm{~min}$ and then sintered again at $500^{\circ} \mathrm{C}$ for 30 min, under dry air flow. To minimize the water absorption from the atmosphere, after cooling down to $150^{\circ} \mathrm{C}$ the substrates were immediately transferred in a nitrogen filled glove box and then let further cooling down to room temperature.

The perovskite films were deposited from a precursor solution containing formamidinium iodide $(1 \mathrm{M})$, lead iodide $(1.1 \mathrm{M})$, methylammonium bromide $(0.2 \mathrm{M})$ and lead bromide $(0.2$ $\mathrm{M})$ in a mixture of anhydrous dimethylformamide : dimethylsulfoxide 4:1 (v:v). The perovskite solution was spin coated using a two steps program at 1000 and $4000 \mathrm{rpm}$ for 10 and $30 \mathrm{~s}$ respectively. During the second step, $100 \mu \mathrm{L}$ of chlorobenzene was poured on the spinning substrate $15 \mathrm{~s}$ prior the end of the program. The substrates were then annealed at $100^{\circ} \mathrm{C}$ for 1 hour in nitrogen filled glove box.

After the perovskite annealing the substrates were cooled down for 1 minute and a spirofluorene linked methoxy triphenylamines (spiro-OMeTAD, from Merck) solution was spun at $4000 \mathrm{rpm}$ for $20 \mathrm{~s}$. The spiro-OMeTAD solutions was prepared in chlorobenzene at concentration of $70 \mathrm{mM}$, and doped with $50 \mathrm{~mol} \%$ of bis(trifluoromethanesulfonyl)imide (LiTFSI, Aldrich) from a stock solution of Li-TFSI $1.8 \mathrm{M}$ in acetonitrile, $330 \mathrm{~mol} \%$ of tertbutylpyridine (Aldrich) and $3 \mathrm{~mol} \%$ of Tris(2-(1H-pyrazol-1-yl)-4-tert-butylpyridine)- 
cobalt(III) Tris(bis(trifluoromethylsulfonyl)imide) (Co-complex, Dyesol) from a stock solution Co-complex $0.25 \mathrm{M}$ in acetonitrile. Finally, $80 \mathrm{~nm}$ of gold was deposited by thermal evaporation under high vacuum, using a shadow masking to pattern the electrodes.

Solar cells characterisation. The solar cells were measured using a $450 \mathrm{~W}$ xenon light source (Oriel). The spectral mismatch between AM1.5G and the simulated illumination was reduced by the use of a Schott K113 Tempax filter (Präzisions Glas \& Optik GmbH). The light intensity was recorded with a Si photodiode equipped with an IR-cutoff filter (KG3, Schott) before each measurement. Current density-voltage (J-V) characteristics of the cells were obtained by applying an external voltage bias while measuring the current response with a digital source meter (Keithley 2400). The cells were masked with a black metal mask $(0.16$ $\mathrm{cm}^{2}$ ) to estimate the active area and reduce the influence of the scattered light. The devices were stored in dry air and dark condition, and they were characterized two days after the perovskite film deposition.

The solar cells current transient dynamics (potentiostatic) and the maximum power point tracking data were measured under 1 sun equivalent white LED illumination with SP300 biologic potentiostat. Maximum point tracking was done with use of a home-developed program, which would keep the devices at the maximum power point by creeping oscillation in voltage and which would measure a full J-V curve every 60 minutes. The devices were placed inside an in house-developed airtight sample holder, which allowed for keeping them under inert, nitrogen atmosphere. Additionally, the backside metal electrode of the devices was placed against a Peltier element, which with a use of a PID controller would keep the actual temperature of the device at $20^{\circ} \mathrm{C}$ regardless the illumination or ambient temperature. Intensity modulated photocurrent spectroscopy was performed using Autolab PGSTAT302N according to procedures previously reported. ${ }^{65}$

\section{Time of flight secondary ion mass spectrometry (ToF-SIMS)}

Samples were prepared with the following configuration: on top of patterned FTO glass, 10 nm of compact $\mathrm{Al}_{2} \mathrm{O}_{3}$ layer was deposited. Following, a $500 \mathrm{~nm}$ layer of $\mathrm{CH}_{3} \mathrm{PbBr}_{3}$ was deposited by spin-coating and $200 \mathrm{~nm}$-thick layer of $\mathrm{PbI}_{2}$ was thermally evaporated. Finally, a $50 \mathrm{~nm}$ layer of $\mathrm{MoO}_{3}$ and $\mathrm{Au}$ electrode were thermally evaporated. Two electronicallyseparated devices (pixels) were fabricated on each substrate. Both pixels were heated to 70 ${ }^{\circ} \mathrm{C}$ under dry air atmosphere and ambient light conditions. One pixel was cycling biased $( \pm 2 \mathrm{~V}$ with 1 hour period) for 16 hours. During the biasing the currents flowing through the pixel 
remained below $1 \mathrm{~mA} \mathrm{~cm}{ }^{-2}$. ToF-SIMS was performed on biased and control pixels according to the method previously reported. ${ }^{13}$

\section{Drift diffusion simulations}

The complete drift diffusion model was published by Richardson et al. elseehwere. ${ }^{34}$ The details of the calculation are reported in the SI. 


\section{Acknowledgements}

A.A. has received funding from the European Union's Seventh Framework Programme for research, technological development and demonstration under grant agreement no 291771. K.D. thanks the SNF for funding within the framework of Umbrella project (Grant Agreement Nos. 407040-153952 and 407040-153990). M.S. acknowledges support from the co-funded Marie Skłodowska Curie fellowship, H2020 Grant agreement no. 665667. N.M. acknowledges support from the BNF program of the University of Bern. Prof. Dr. Hans J. Hug, Dr. Laetitia Bernard and Prof. Dr. Arnaud Delcorte are gratefully acknowledged, respectively for N.M. EMPA affiliation and access to the ToF-SIMS software. U.S., A.A. and B.R. thank the Adolphe Merkle Foundation and the NRP 70 Energy Turnaround - SNF. 


\section{References}

1. J. Gong, S. B. Darling and F. You, Energy \& Environmental Science, 2015, 8, 1953-1968.

2. NationalRenewableEnergyLaboratory, Best research-cell efficiencies, http://www.nrel.gov/ncpv/images/efficiency_chart.jpg).

3. A. Abate, S. Paek, F. Giordano, J.-P. Correa-Baena, M. Saliba, P. Gao, T. Matsui, J. Ko, S. M. Zakeeruddin and K. H. Dahmen, Energy \& Environmental Science, 2015, 8, 2946-2953.

4. J. You, L. Meng, T.-B. Song, T.-F. Guo, Y. M. Yang, W.-H. Chang, Z. Hong, H. Chen, H. Zhou and Q. Chen, Nature nanotechnology, 2016, 11, 75-81.

5. Z. Song, A. Abate, S. C. Watthage, G. K. Liyanage, A. B. Phillips, U. Steiner, M. Graetzel and M. J. Heben, Advanced Energy Materials, 2016.

6. R. J. Sutton, G. E. Eperon, L. Miranda, E. S. Parrott, B. A. Kamino, J. B. Patel, M. T. Hörantner, M. B. Johnston, A. A. Haghighirad and D. T. Moore, Advanced Energy Materials, 2016.

7. M. Saliba, T. Matsui, J.-Y. Seo, K. Domanski, J.-P. Correa-Baena, M. K. Nazeeruddin, S. M. Zakeeruddin, W. Tress, A. Abate and A. Hagfeldt, Energy \& Environmental Science, 2016, 9, 1989-1997.

8. D. Bryant, N. Aristidou, S. Pont, I. Sanchez-Molina, T. Chotchunangatchaval, S. Wheeler, J. R. Durrant and S. A. Haque, Energy \& Environmental Science, 2016, 9, 1655-1660.

9. B. Roose, J. P. Correa Baena, K. C. Gödel, M. Graetzel, A. Hagfeldt, U. Steiner and A. Abate, Nano Energy, 2016.

10. F. Bella, G. Griffini, J.-P. Correa-Baena, G. Saracco, M. Grätzel, A. Hagfeldt, S. Turri and C. Gerbaldi, Science, 2016, aah4046.

11. T. J. Wilderspin, F. De Rossi and T. M. Watson, Solar Energy, 2016, 139, 426-432.

12. A. Calloni, A. Abate, G. Bussetti, G. Berti, R. Yivlialin, F. Ciccacci and L. Duò, The Journal of Physical Chemistry C, 2015, 119, 21329-21335.

13. K. Domanski, J.-P. Correa-Baena, N. Mine, M. K. Nazeeruddin, A. Abate, M. Saliba, W. Tress, A. Hagfeldt and M. Grätzel, ACS nano, 2016.

14. A. Mei, X. Li, L. Liu, Z. Ku, T. Liu, Y. Rong, M. Xu, M. Hu, J. Chen and Y. Yang, Science, 2014, 345, 295-298.

15. K. A. Bush, C. D. Bailie, Y. Chen, A. R. Bowring, W. Wang, W. Ma, T. Leijtens, F. Moghadam and M. D. McGehee, Advanced Materials, 2016.

16. S. Guarnera, A. Abate, W. Zhang, J. M. Foster, G. Richardson, A. Petrozza and H. J. Snaith, The journal of physical chemistry letters, 2015, 6, 432-437.

17. B. Roose, K. C. Gödel, S. Pathak, A. Sadhanala, J. P. C. Baena, B. D. Wilts, H. J. Snaith, U. Wiesner, M. Grätzel and U. Steiner, Advanced Energy Materials, 2016, 6.

18. M. Saliba, T. Matsui, K. Domanski, J.-Y. Seo, A. Ummadisingu, S. M. Zakeeruddin, J.-P. CorreaBaena, W. R. Tress, A. Abate and A. Hagfeldt, Science, 2016, 354, 206-209.

19. Z. Xiao, Y. Yuan, Y. Shao, Q. Wang, Q. Dong, C. Bi, P. Sharma, A. Gruverman and J. Huang, Nature materials, 2015, 14, 193-198.

20. Y. Yuan and J. Huang, Accounts of chemical research, 2016, 49, 286-293.

21. Y. Shao, Y. Fang, T. Li, Q. Wang, Q. Dong, Y. Deng, Y. Yuan, H. Wei, M. Wang and A. Gruverman, Energy \& Environmental Science, 2016, 9, 1752-1759.

22. Y. Zou and R. J. Holmes, Advanced Energy Materials, 2016.

23. W.-J. Yin, T. Shi and Y. Yan, Applied Physics Letters, 2014, 104, 063903.

24. A. Buin, R. Comin, J. Xu, A. H. Ip and E. H. Sargent, Chemistry of Materials, 2015, 27, 44054412.

25. J. M. Azpiroz, E. Mosconi, J. Bisquert and F. De Angelis, Energy \& Environmental Science, 2015, 8, 2118-2127.

26. C. Eames, J. M. Frost, P. R. Barnes, B. C. O'regan, A. Walsh and M. S. Islam, Nature communications, 2015, 6. 
27. J. Haruyama, K. Sodeyama, L. Han and Y. Tateyama, Journal of the American Chemical Society, 2015, 137, 10048-10051.

28. V. W. Bergmann, Y. Guo, H. Tanaka, I. M. Hermes, D. Li, A. Klasen, S. A. Bretschneider, E. Nakamura, R. Berger and S. A. Weber, ACS Applied Materials \& Interfaces, 2016.

29. R. Cao, F. Xu, J. Zhu, S. Ge, W. Wang, H. Xu, R. Xu, Y. Wu, Z. Ma and F. Hong, Advanced Energy Materials, 2016.

30. S. Chen, X. Wen, R. Sheng, S. Huang, X. Deng, M. A. Green and A. Ho-Baillie, ACS applied materials \& interfaces, 2016, 8, 5351-5357.

31. P. Calado, A. M. Telford, D. Bryant, X. Li, J. Nelson, B. C. O'Regan and P. R. Barnes, arXiv preprint arXiv:1606.00818, 2016.

32. O. Hentz, Z. Zhao and S. Gradecak, Nano letters, 2016, 16, 1485-1490.

33. I. Zarazua, J. Bisquert and G. Garcia-Belmonte, The journal of physical chemistry letters, 2016, 7, 525-528.

34. G. Richardson, S. E. O'Kane, R. G. Niemann, T. A. Peltola, J. M. Foster, P. J. Cameron and A. B. Walker, Energy \& Environmental Science, 2016, 9, 1476-1485.

35. S. D. Stranks, V. M. Burlakov, T. Leijtens, J. M. Ball, A. Goriely and H. J. Snaith, Physical Review Applied, 2014, 2, 034007.

36. W. Tress, N. Marinova, T. Moehl, S. Zakeeruddin, M. K. Nazeeruddin and M. Grätzel, Energy \& Environmental Science, 2015, 8, 995-1004.

37. W. Tress, J. P. Correa Baena, M. Saliba, A. Abate and M. Graetzel, Advanced Energy Materials, 2016.

38. S. van Reenen, M. Kemerink and H. J. Snaith, The journal of physical chemistry letters, 2015, 6, 3808-3814.

39. J. Carrillo, A. Guerrero, S. Rahimnejad, O. Almora, I. Zarazua, E. Mas-Marza, J. Bisquert and G. Garcia-Belmonte, Advanced Energy Materials, 2016, 6.

40. H. Yu, H. Lu, F. Xie, S. Zhou and N. Zhao, Advanced Functional Materials, 2016.

41. S. Meloni, T. Moehl, W. Tress, M. Franckevičius, M. Saliba, Y. H. Lee, P. Gao, M. K. Nazeeruddin, S. M. Zakeeruddin and U. Rothlisberger, Nature communications, 2016, 7.

42. T. S. Ripolles, A. K. Baranwal, K. Nishinaka, Y. Ogomi, G. Garcia-Belmonte and S. Hayase, Physical Chemistry Chemical Physics, 2016, 18, 14970-14975.

43. O. Almora, C. Aranda, I. Zarazua, A. Guerrero and G. Garcia-Belmonte, ACS Energy Letters, 2016.

44. H. J. Snaith, A. Abate, J. M. Ball, G. E. Eperon, T. Leijtens, N. K. Noel, S. D. Stranks, J. T.-W. Wang, K. Wojciechowski and W. Zhang, The journal of physical chemistry letters, 2014, 5, 1511-1515.

45. E. Unger, E. Hoke, C. Bailie, W. Nguyen, A. Bowring, T. Heumüller, M. Christoforo and M. McGehee, Energy \& Environmental Science, 2014, 7, 3690-3698.

46. I. Levine, P. K. Nayak, J. T.-W. Wang, N. Sakai, S. Van Reenen, T. M. Brenner, S. Mukhopadhyay, H. J. Snaith, G. Hodes and D. Cahen, arXiv preprint arXiv:1604.03907, 2016.

47. T. Zhang, H. Chen, Y. Bai, S. Xiao, L. Zhu, C. Hu, Q. Xue and S. Yang, Nano Energy, 2016.

48. Y. Zhao, W. Zhou, W. Ma, S. Meng, H. Li, J. Wei, R. Fu, K. Liu, D. Yu and Q. Zhao, ACS Energy Letters, 2016, 1, 266-272.

49. K. C. Kwon, K. Hong, Q. Van Le, S. Y. Lee, J. Choi, K. B. Kim, S. Y. Kim and H. W. Jang, Advanced Functional Materials, 2016.

50. O. Almora, A. Guerrero and G. Garcia-Belmonte, Applied Physics Letters, 2016, 108, 043903.

51. S. Bae, S. Kim, S.-W. Lee, K. Cho, S. Park, S. Lee, Y. Kang, H.-S. Lee and D. Kim, The Journal of Physical Chemistry Letters, 2016.

52. M. Bag, L. A. Renna, R. Y. Adhikari, S. Karak, F. Liu, P. M. Lahti, T. P. Russell, M. T. Tuominen and D. Venkataraman, Journal of the American Chemical Society, 2015, 137, 13130-13137.

53. F. Huang, L. Jiang, A. R. Pascoe, Y. Yan, U. Bach, L. Spiccia and Y.-B. Cheng, Nano Energy, 2016. 
54. W. Nie, J.-C. Blancon, A. J. Neukirch, K. Appavoo, H. Tsai, M. Chhowalla, M. A. Alam, M. Y. Sfeir, C. Katan and J. Even, Nature communications, 2016, 7.

55. J. S. Yun, J. Seidel, J. Kim, A. M. Soufiani, S. Huang, J. Lau, N. J. Jeon, S. I. Seok, M. A. Green and A. Ho-Baillie, Advanced Energy Materials, 2016.

56. N. J. Jeon, J. H. Noh, Y. C. Kim, W. S. Yang, S. Ryu and S. I. Seok, Nature materials, 2014, 13, 897-903.

57. X. Zhao and N.-G. Park, 2015.

58. E. H. Anaraki, A. Kermanpur, L. Steier, K. Domanski, T. Matsui, W. Tress, M. Saliba, A. Abate, M. Grätzel and A. Hagfeldt, Energy \& Environmental Science, 2016, 9, 3128-3134.

59. A. M. Belu, D. J. Graham and D. G. Castner, Biomaterials, 2003, 24, 3635-3653.

60. C. Li, S. Tscheuschner, F. Paulus, P. E. Hopkinson, J. Kießling, A. Köhler, Y. Vaynzof and S. Huettner, Advanced Materials, 2016.

61. D. Li, H. Wu, H.-C. Cheng, G. Wang, Y. Huang and X. Duan, ACS nano, 2016, 10, 6933-6941.

62. S. J. Yoon, S. Draguta, J. S. Manser, O. Sharia, W. F. Schneider, M. Kuno and P. V. Kamat, ACS Energy Letters, 2016, 1, 290-296.

63. E. Guillén, F. J. Ramos, J. A. Anta and S. Ahmad, The Journal of Physical Chemistry C, 2014, 118, 22913-22922.

64. J. Y. Seo, T. Matsui, J. Luo, J. P. Correa-Baena, F. Giordano, M. Saliba, K. Schenk, A. Ummadisingu, K. Domanski and M. Hadadian, Advanced Energy Materials, 2016.

65. J. P. Correa-Baena, M. Anaya, G. Lozano, W. Tress, K. Domanski, M. Saliba, T. Matsui, T. J. Jacobsson, M. E. Calvo and A. Abate, Advanced Materials, 2016.

66. E. Mosconi and F. De Angelis, ACS Energy Letters, 2016.

67. D. Yang, W. Ming, H. Shi, L. Zhang and M.-H. Du, Chemistry of Materials, 2016.

68. W. Ming, S. Chen and M.-H. Du, Journal of Materials Chemistry A, 2016, 4, 16975-16981.

69. A. Walsh, D. O. Scanlon, S. Chen, X. Gong and S. H. Wei, Angewandte Chemie, 2015, 127, 1811-1814. 